\title{
Special Focus on Two-Dimensional Materials and Device Applications*
}

The exploration of two dimensional layered materials (2DLMs) with atomic thickness and rich bandstructures has taken both the scientific and technological communities by storm during the past one and a half decade. Graphene plays a pioneering role in the development of 2DLMs but its gapless bandstructure somehow limits further applications. Following the huge success of graphene, more and more 2DLMs have been discovered and studied, including the transition metal dichalcogenides (TMDs), hexagonal boron nitride (h-BN), black phosphorus (BP), silicene, 2D perovskites, etc. So far, the fast pace of TMD material development shows no sign of slow down. More research topics have been systematically explored in various types of $2 \mathrm{DLM}$, such as quantum transport, spintronics, topological phases, $2 \mathrm{D}$ magnetism and $2 \mathrm{D}$ ferroelectricity.

Despite the continuous exciting achievements of 2DLMs, challenges still exist. The main bottleneck preventing practical applications is apparently the large-scale and uniform synthesis of 2DLM with high quality and controlled structure. On the other hand, until now most 2DLMs still remain a platform for fundamental research. Regarding practical application of 2DLMs, it is difficult yet unrealistic to use them to replace certain types of conventional materials in a short period, such as the efforts to replace Si-based electronics. However, 2DLMs can certainly find their places in some appropriate applications such as flexible and low-power devices that more directly benefit from their "atomically thin nature". Also, with the nonstop efforts from emerging interdisciplinary research fields, we believe the unique properties of 2DLMs will definitely inspire new applications in the near future. Targeting at the above goals, this special focus on two-dimensional materials and device applications of SCIENCE CHINA Information Sciences attempts to present recent works related to 2DLMs, covering a wide array of topics.

Transition metal dichalcogenides (TMDs) exhibit versatile bandstructures and thus can be utilized in various applications from logic to optoelectronic devices. Tang et al. review the recent developments of wafer-scale TMDs film from material synthesis to device and circuits application. For the synthesis of continuous TMD films, chemical vapor deposition method is mainly discussed, providing a scalable and controllable route for the growth of high-quality films. Basic logic units and other circuits fabricated in large scale are summarized. In addition, memory, optoelectronic and analog devices fabricated on wafer-scale TMD films are also included in their review.

Zhang et al. review the latest progress on the topic of $2 \mathrm{D}$ ferroic orderings from both experimental and theoretical points of view. This review mainly focuses on two portions: $2 \mathrm{D}$ magnetism and 2D ferroelectricity. The booming of ferroic orderings in van der Waals 2DLMs are introduced and summarized in the review. It also discusses their tremendous opportunities in the development of fundamental physics and potential information industrial applications.

Qin et al. review the recent progress in synthesis of carbon nanotube, graphene and their hybrid architectures, together with the application in the electronic, optoelectronic devices and supercapacitors based on all-carbon hybrids. They show that all-carbon hybrids could be one of the most promising

* Citation Wang X R, Zhou P. Special focus on two-dimensional materials and device applications. Sci China Inf Sci, 2019, 62(12): 220400, https://doi.org/10.1007/s11432-019-2696-1 
strategies to achieve high-performance devices. All-carbon hybrids based electronic, optoelectronic, and energy-storage devices are discussed, such as logic inverters, optoelectronic memory, electroluminescent light emitters, photodetectors and supercapacitors. Finally, they discuss the challenges of scalable applications and provide perspectives on the opportunities for future application.

Yang et al. report a nonvolatile memory based on In2Se3-Wse2 vdW ferroelectric heterostructure. The high-quality In2Se3 with single crystallinity is synthesized by chemical vapor transport method and exhibits stable out-of-plane ferroelectricity at room temperature. Upon assembling In2Se3/WSe2 vertical heterostructures, switchable diode effect and nonvolatile memory phenomenon are observed. This type of device provides a novel way for exploring $2 \mathrm{D}$ ferroelectrics material in the application of information storage.

$\mathrm{Wu}$ et al. explored the optoelectronic properties of MoS2 transistor through electrolyte gating. The experiment results show that electrolyte provides a strong gate control in the MoS2 transistor, and Schottky barrier between MoS2 and contact electrodes can be effectively modulated from 11 to $179 \mathrm{meV}$. The photo-mapping result indicates the photocurrent is mainly generated in the Schottky junction region. Such device exhibits a high responsivity of $2.68 \times 104 \mathrm{~A} / \mathrm{W}$ and detectivity of $9.6 \times 1010$ Jones, indicates that electrolyte gating can improve the optoelectronic sensitivity of MoS2 phototransistor.

Wang et al. first demonstrate a pulse output using a VSe2-based passively Q-switched laser. The high-quality VSe2 saturable absorber, synthesized by liquid-phase exfoliation method, had a saturated intensity of $533.8 \mathrm{~J} / \mathrm{cm} 2$ and modulation depth of 9.9

Two-dimensional transition metal oxychlorides ( $\mathrm{MOCl}, \mathrm{M}=\mathrm{Fe}, \mathrm{Cr}, \mathrm{V}, \mathrm{Ti}, \mathrm{Sc}$ ) with metal-oxygen plane sandwiched by two layers of chloride ions possess a lot of novel material properties. Yan et al. synthesized freestanding VOCl flakes with various thicknesses through CVD method and demonstrated their application in electronic devices. The obtained VOCl-based memristive devices show low power consumption and excellent device reliability. The memristive devices are also used to emulate the depression and potentiation behaviors of synaptic plasticity.

Guest Editors:

Xinran WANG

Nanjing University

Peng ZHOU

Fudan University 